\title{
Immune response to SARS sets up puzzling paradox
}

Severe acute respiratory syndrome (SARS) rarely makes headlines these days, but immunologists are still chasing down why only a subset of people became severely ill and died from the disease. Popular hypotheses all suggest that an overactive or otherwise dysfunctional immune response may be to blame.

SARS last year claimed nearly 800 lives worldwide. Many researchers initially suggested that those who die have an overactive immune system that triggers a destructive inflammatory response.

The theory was supported by anecdotal reports that at a hospital in Guangzhou, the southern Chinese city where SARS originated, AIDS patients-who presumably have weak immune systems-were resistant to SARS infection.

Scientists were also intrigued that children, prime candidates for most respiratory illnesses, seemed to escape the brunt of the epidemic. "The whole mechanism of disease causation in SARS is quite an enigma," says Malik Peiris, professor of microbiology at Hong Kong University.

Several teams are now trying to solve the puzzle at a molecular level, but have had to rely on animal models and samples from previous outbreaks. "Everyone wants a glimpse of the

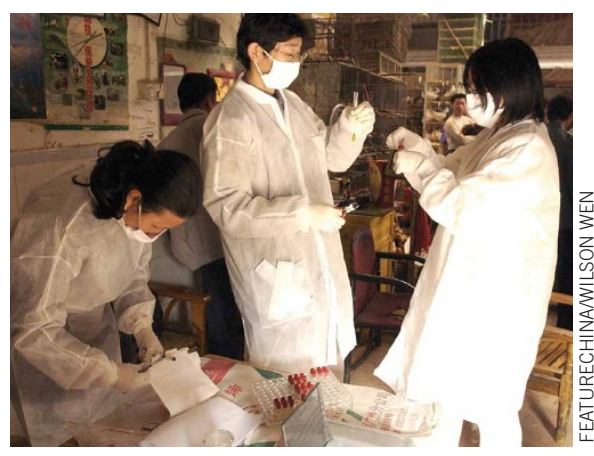

Scientists are baffled by how SARS, which claimed nearly 800 lives last year, affects the immune system.

immune response in SARS patients," says David Kelvin, head of experimental therapeutics at University Health Network in Toronto.

Kelvin and his colleagues collected samples from 110 patients - 35 of them within the first 24 hours after symptoms appeared-and followed them approximately every five days; 45 patients were tracked for more than a year. The researchers looked at gene expression profiles of various immune cells and markers to study both the immediate, or innate, immune response and the later adaptive immune response.

Based on their observations, Kelvin says, those who become severely ill are predisposed either by genetics or by their immune history to be particularly susceptible. "We think they're lacking an arm of the immune system," he says. He declined to reveal further details because the data are unpublished. The researchers are also collaborating with a Taiwanese team to identify a genetic predisposition to SARS.

Others are still pursuing the idea of an overactive immune response. Haichao Wang and his colleagues are investigating the role of HMGB1, a late-stage cytokine that mediates lung injury and inflammation. Wang, chief of basic science research at the North Shore University Hospital in New York, is testing the effect of Chinese herbal recipes used as SARS treatments during the outbreaks. He has found preliminary evidence that some of the herbs can block HMGB1 and prevent the toxic inflammatory cascade seen in SARS.

Peiris is also following molecular leads on the immune response to SARS, but declined to reveal details. "We still don't have an exact idea of what the immune system is doing," he says. "Maybe it's not functioning well or maybe it's functioning too well."

Apoorva Mandavilli, New York With additional reporting by David Cyranoski

\section{HIV vaccine research a shot in the arm for immunologists}

Decades of research have failed to produce a slam-dunk AIDS vaccine, but the fruitless efforts have proven a boon for basic immunologists, adding new tools and knowledge to their repertoire.

Most of these gains derive from something that initially stymied HIV vaccine researchers: animal models don't accurately predict which AIDS vaccine will work in people. Rather than test their candidates in mice and nonhuman primates, researchers were forced to assess them in people.

Because of this, "there's been an explosion in the field of human immunology," says Rick Koup, chief of immunology at the US National Institutes of Health's Vaccine Research Center. "Before, the vast majority of immunology research was done in mice, and there was almost no strong basic immunology work going on in either humans or nonhuman primates," Koup says.

When scientists began studying human immunology, they realized the picture was much more complicated than previously thought. This first became apparent when HIV vaccine researchers tried to mimic traditional vaccines by injecting proteins from the coat of the HIV virus. It didn't work.

The first HIV vaccines stimulated antibodies, just like more traditional vaccines. But the antibodies generated by the first AIDS vaccines did not prevent the virus from infecting people, or from progressing after the initial infection.

Scientists then began working on an HIV vaccine that could stimulate robust cellular response in addition to the strong antibody response. But even vaccines that invoke a strong cellular response did not protect people from HIV infection. This stumbling block has led to another breakthrough in understanding of the immune system: the cellular immune response to vaccines is itself quite nuanced.

Scientists have since realized that chronic infections, such as HIV, interact with the immune system in a completely different way than do acute infections. For instance, Giuseppe Pantaleo and coworkers have found that the human CD8 T-cell response to HIV infection is skewed so that less protective cells dominate (Nature 410, 106;
2001). His group has also shown that HIVpositive people who stay relatively healthy over a long time have a more diverse helper T-cell repertoire than those who rapidly become ill (Blood 103, 966; 2003).

Eliciting the right balance of T-cell subpopulations is now a main goal in HIV vaccine research. Scientists are aided by the development of new technologies, such as flow cytometry, that allow the indepth study of the immune system, and that were developed in part by the HIV vaccine field.

By examining why HIV vaccines have failed, researchers have also learned more about the immune factors-dubbed 'correlates of protection' - that make any vaccine work. That has led to the idea of rational vaccine design, in which researchers try to engineer a vaccine to elicit a particular protective response. If it works, this idea of protection by design - rather than by a lucky guess-may be the HIV vaccine field's most lasting legacy for immunology.

Erika Check, Washington, DC 